\title{
Stability of a Delayed SIQRS Model with Temporary Immunity
}

\author{
Laid Chahrazed, Rahmani Fouad Lazhar \\ Department of Mathematics, Faculty of Exact Sciences, University Constantine 1, Constantine, Algeria \\ Email: Chahrazed2009@yahoo.fr
}

Received September 7, 2012; revised December 1, 2012; accepted December 16, 2012

\begin{abstract}
This paper addresses a time-delayed SIQRS model with a linear incidence rate. Immunity gained by experiencing the disease is temporary; whenever infected, the disease individuals will return to the susceptible class after a fixed period of time. First, the local and global stabilities of the infection-free equilibrium are analyzed, respectively. Second, the endemic equilibrium is formulated in terms of the incidence rate, and locally asymptotic stability. Finally we use the adomian decomposition method is applied to the system epidemiologic. This method yields an analytical solution in terms of convergent infinite power series.
\end{abstract}

Keywords: Adomian Method; Epidemiology; Mathematical Model; The Epidemic Model; The Equilibrium Points

\section{Introduction}

In the past, the epidemiology is restricted to the study of morbid phenomena resulting in an increase in sudden sharp and localized in space, the number of cases and time. It focuses primarily on infectious diseases. Epidemiology is a set of methods research by conducting investigations and a decision tool. Infectious diseases are one area where the theoretical were more developed in epidemiology. The mathematical theory of epidemics provides a framework for reconstruction history of past pandemics, contributing to a better understanding transmission mechanisms, a warning earlier vis-à-vis emergent phenomena, and now the prediction of epidemic spread in time and space. Generally, a model contains a disease-free equilibrium and one or multiple equilibria are endemic. The stability of a disease-free status equilibrium and the existence of other nontrivial equilibria can be determined by the ratio called the basic reproductive number, which quantifies the number of secondary infections arise from a simply put infected in a population of sensitive. When the basic reproduction number is less than unity, disease-free equilibrium is locally asymptotically stable, and therefore the disease off after a certain period of time. Similarly, when endemic equilibrium is global attractor, epidemiologically, it means that the disease will prevail and persist in a population and overall stability of these models is relatively low, especially models with delays.

In this paper, we discuss the equilibrium and stability of the model SIQR epidemic with constant infectious period which is made of a delay time. A particular assumption is made that the time which individuals remain infectious can be described by an exponential distribution. This distribution corresponds to the assumption that the chances of recovery in a given time interval are independent of time since infection. To solve the system is the autile decomposition method and for this we use the references of [1-14]. In order to describe the effects of disease immunity temporal delays are often incorporated in such models [15-19].

\section{Model Equations}

The system is described by equations which are defined as follows:

$$
\left\{\begin{array}{l}
\dot{S}(t)=\mu-\mu_{1} S(t)-\alpha S(t) I(t)+\gamma I(t-\tau) \mathrm{e}^{-\mu_{4} \tau}, \\
\dot{I}(t)=\alpha S(t) I(t)-\left(\mu_{2}+\beta+\gamma\right) I(t), \\
\dot{Q}(t)=\beta I(t)-\left(\rho+\mu_{3}\right) Q(t), \\
\dot{R}(t)=\rho Q(t)+\gamma I(t)-\mu_{4} R(t)-\gamma I(t-\tau) \mathrm{e}^{-\mu_{4} \tau} .
\end{array}\right.
$$

D'où $S(t)+I(t)+Q(t)+R(t)=N(t)$ denotes the population size at time $t ; S(t), I(t), Q(t)$ and $R(t)$ denote the sizes of the population susceptible to disease, and infectious members, quarantine members and those who were removed from the possibility of infection through temporary immunity, respectively. It is assumed that all new borns are susceptible.

The positive constants $\mu_{1}, \mu_{2}, \mu_{3}$ and $\mu_{4}$ represent the death rates of susceptible, infectious and those who are being quarantiane and recovered, respectively. Biolo- 
gically, it is natural to assume that $\mu_{1} \leq \min \left\{\mu_{2}, \mu_{3}, \mu_{4}\right\}$. The positive constants $\mu$ and $\gamma$ represent the birth rate (from insidence) of the population and the recovery rate of infection, respectively. The positive constant $\alpha$ is the average number of contacts per infective. The positive constants $\beta, \rho$ are the numbers of transfers or conversions of infected people quarantined and quarantined at recovered. The term $\gamma I(t-\tau) \mathrm{e}^{-\mu_{4} \tau}$ indicates that an individual has survived to natural death in a pool recovery before becoming susceptible again, where $\tau$ is the length of immunity period.

The initial condition of (1) is given as:

$$
\begin{aligned}
& S(\eta)=\Phi_{1}(\eta), I(\eta)=\Phi_{2}(\eta), Q(\eta)=\Phi_{3}(\eta), \\
& R(\eta)=\Phi_{4}(\eta),-\tau \leq \eta \leq 0,
\end{aligned}
$$

where $\Phi=\left(\Phi_{1}, \Phi_{2}, \Phi_{3}, \Phi_{4}\right)^{\mathrm{T}} \in \mathbb{C}$ such that

$$
\begin{aligned}
& S(\eta)=\Phi_{1}(\eta)=\Phi_{1}(0) \geq 0, I(\eta)=\Phi_{2}(\eta)=\Phi_{2}(0) \geq 0, \\
& Q(\eta)=\Phi_{3}(\eta)=\Phi_{3}(0) \geq 0, R(\eta)=\Phi_{4}(\eta)=\Phi_{4}(0) \geq 0 .
\end{aligned}
$$

We have $C$ denote the Banach space $C\left([-\tau, 0], \mathbb{R}^{4}\right)$ of continuous functions mapping the interval $[-\tau, 0]$ into $\mathbb{R}^{4}$. By a biological meaning, we further assume that $\Phi_{1}(\eta)=\Phi_{1}(0) \geq 0$ for $i=1,2,3,4$.

Since $R(t)$ does not appear explicitly in the first three equations of (1), instead of (1) we consider the system:

$$
\left\{\begin{array}{l}
\dot{S}(t)=\mu-\mu_{1} S(t)-\alpha S(t) I(t)+\gamma I(t-\tau) \mathrm{e}^{-\mu_{4} \tau}, \\
\dot{I}(t)=\alpha S(t) I(t)-\left(\mu_{2}+\beta+\gamma\right) I(t), \\
\dot{Q}(t)=\beta I(t)-\left(\rho+\mu_{3}\right) Q(t) .
\end{array}\right.
$$

With the initial condition

$$
\begin{aligned}
& S(\eta)=\Phi_{1}(\eta), I(\eta)=\Phi_{2}(\eta), \\
& Q(\eta)=\Phi_{3}(\eta),-\tau \leq \eta \leq 0,
\end{aligned}
$$

where, $\Phi_{1}(0) \geq 0, \Phi_{2}(0) \geq 0, \Phi_{3}(0) \geq 0,-\tau \leq \eta \leq 0$. we obtain $\dot{N}(t) \leq \mu-\mu_{1} N(t)$, and $S(t)+I(t)+Q(t) \leq N(t)$.

The region $\Omega=\left\{(S, I, Q) \in \mathbb{R}_{+}^{3}, S+I+Q \leq N \prec \frac{\mu}{\mu_{1}}\right\}$ is positively invariant set of (3).

\section{The Disease Free Equilibrium and Its Stability}

An equilibrium point of system (3) satisfies:

$$
\left\{\begin{array}{l}
\mu-\mu_{1} S-\alpha S I+\gamma I \mathrm{e}^{-\mu_{4} \tau}=0, \\
\alpha S I-\left(\mu_{2}+\beta+\gamma\right) I=0, \\
\beta I-\left(\rho+\mu_{3}\right) Q=0 .
\end{array}\right.
$$

We calculate the points of equilibria in the absence and presence of infection.
In the absence of infection $I=0$, substituting in the system we obtain the first equilibrium point:

$$
P_{0}=(\hat{S}, \hat{I}, \hat{Q})^{\mathrm{T}}=\left(\frac{\mu}{\mu_{1}}, 0,0\right)^{\mathrm{T}}
$$

We calculate the Jacobian matrix according to the system (3) with $P_{0}$.

$$
J\left(P_{0}\right)=\left[\begin{array}{ccc}
-\mu_{1} & -\alpha \frac{\mu}{\mu_{1}}+\gamma \mathrm{e}^{-\mu_{4} \tau} & 0 \\
0 & \alpha \frac{\mu}{\mu_{1}}-\left(\mu_{2}+\beta+\gamma\right) & 0 \\
0 & \beta & -\left(\rho+\mu_{3}\right)
\end{array}\right]
$$

The epidemic is locally asymptotically stable if and only if all eigenvalues of the Jacobian matrix $\mathrm{J}$ have negative real part. The eigenvalues can be determined by solving the characteristic equation.

$$
\operatorname{det}\left(\begin{array}{ccc}
-\mu_{1}-\lambda & -\frac{\alpha \mu}{\mu_{1}}+\gamma \mathrm{e}^{-\mu_{4} \tau} & 0 \\
0 & \frac{\alpha \mu}{\mu_{1}}-\left(\mu_{2}+\beta+\gamma\right)-\lambda & 0 \\
0 & \beta & -\left(\rho+\mu_{3}\right)-\lambda
\end{array}\right)
$$

So the three eigenvalues are:

$$
\lambda_{1}=-\mu_{1}, \lambda_{2}=\frac{\alpha \mu}{\mu_{1}}-\left(\mu_{2}+\beta+\gamma\right), \lambda_{3}=-\left(\rho+\mu_{3}\right)
$$

In order for $\lambda_{1}, \lambda_{2}$, and $\lambda_{3}$ to be negative, it is required that

$$
\frac{\alpha \mu}{\mu_{1}} \prec\left(\mu_{2}+\beta+\gamma\right)
$$

Then we define the basic reproduction number of the infection $R_{0}$ as follows

$$
R_{0}=\frac{\alpha \mu}{\mu_{1\left(\mu_{2}+\beta+\gamma\right)}} .
$$

\subsection{Stability of Equilibrium Point $P_{0}$}

The basic reproduction number $R_{0}$ is defined as the total number of infected population in the resulting subinfected population where almost all of the uninfected.

Theorem 1. The disease-free equilibrium $P_{0}$ is locally asymptotically stable if $R_{0}<1$ and unstable if $R_{0}>1$.

\subsection{Existence of Endemic Equilibrium and Its Locally Asymptotical Stability}

From the previous section it is follows that when the 
trivial equilibrium $P_{0}$ of system (3) is locally asymptotically stable, then endemic equilibrium does not exist. When $R_{0}>1$, system (3) has a unique non-trivial equilibrium $P_{\tau}^{*}$ other than the disease-free equilibrium.

In the presence of infection $I \neq 0$, substituting in the system we obtain the second equilibrium point:

$$
\begin{aligned}
& P_{\tau}^{*}=\left(S_{\tau}^{*}, I_{\tau}^{*}, Q_{\tau}^{*}\right)^{\mathrm{T}} \\
& =\left(\frac{\mu_{2}+\beta+\gamma}{\alpha}, \frac{\alpha \mu-\mu_{1}\left(\mu_{2}+\beta+\gamma\right)}{\alpha\left(\left(\mu_{2}+\beta\right)+\gamma\left(1-\mathrm{e}^{-\mu_{4} \tau}\right)\right)}, \frac{\rho+\mu_{3}}{\beta} I_{\tau}^{*}\right)^{\mathrm{T}}
\end{aligned}
$$

Using the simplified and intuitive point theorem, Lya- punov square near the fixed point, and the solutions of the nonlinear system associated with the linear system by applying the Taylor formula of order 1 .

Let

$$
h(t)=S(t)-S_{\tau}^{*}, k(t)=I(t)-I_{\tau}^{*}, m(t)=Q(t)-Q_{\tau}^{*},
$$

we note $h, k$, and $m$ are the small perturbations. The formula for the Taylor series expansion.

We calculate the Jacobian matrix according to the system (3) with $P_{\tau}^{*}$. The epidemic is locally asymptotically stable if and only if all eigenvaluesof the Jacobian matrix J have negative real part. The eigenvalues can be determined by solving the characteristic equation.

$$
\operatorname{det}\left(\begin{array}{ccc}
-\mu_{1}-\alpha I_{\tau}^{*}-\lambda & -\alpha S_{\tau}^{*}+\gamma \mathrm{e}^{-\mu_{4} \tau} & 0 \\
\alpha I_{\tau}^{*} & -\lambda & 0 \\
0 & \beta & -\left(\rho+\mu_{3}\right)-\lambda
\end{array}\right)=0 .
$$

Since $\tau=0$, we have

$$
P_{\tau}^{*}=\left(S_{\tau}^{*}, I_{\tau}^{*}, Q_{\tau}^{*}\right)^{\mathrm{T}}=\left(\frac{\mu_{2}+\beta+\gamma}{\alpha}, \frac{\alpha \mu-\mu_{1}\left(\mu_{2}+\beta+\gamma\right)}{\alpha\left(\mu_{2}+\beta\right)}, \frac{\rho+\mu_{3}}{\beta} I_{0}^{*}\right)^{\mathrm{T}} .
$$

Then (10) is then written:

$$
\operatorname{det}\left(\begin{array}{ccc}
\left(\frac{-\alpha \mu-\mu_{1} \gamma}{\mu_{2}+\beta}\right)-\lambda & -\left(\mu_{2}+\beta+\gamma\right) & 0 \\
\frac{\alpha \mu-\mu_{1}\left(\mu_{2}+\beta+\gamma\right)}{\mu_{2}+\beta} & -\lambda & 0 \\
0 & \beta & -\left(\rho+\mu_{3}\right)-\lambda
\end{array}\right)=0 .
$$

The caracteristic equation is as follow:

$$
\lambda^{3}+a \lambda^{2}+b \lambda+c=0
$$

with the notations:

$$
\begin{aligned}
& a=\frac{\alpha \mu+\mu_{1} \gamma}{\mu_{2}+\beta}+\left(\rho+\mu_{3}\right), \\
& b=\frac{\left(\mu_{2}+\beta+\gamma\right)\left(\alpha \mu-\mu_{1}\left(\mu_{2}+\beta+\gamma\right)\right)-\left(\rho+\mu_{3}\right)\left(-\alpha \mu-\mu_{1} \gamma\right)}{\mu_{2}+\beta}, \\
& c=\frac{\left(\rho+\mu_{3}\right)\left(\mu_{2}+\beta+\gamma\right)\left[\alpha \mu-\mu_{1}\left(\mu_{2}+\beta+\gamma\right)\right]}{\mu_{2}+\beta} .
\end{aligned}
$$

When $R_{0}>1$ we have $a>0, b>0$, and $c>0$. The real parts of eigenvalues are négative, then the equilibrium $P_{\tau}^{*}$ is locally asymptotically stable.

With $R_{0}>1$, system (3) has a unique non-trivial equilibrium $P_{\tau}^{*}$ is locally asymptotically stable.

\section{The Adomian Decomposition Method}

The Adomian decomposition method has been applied to broad classes of problems in many fields such as mathematics, physics, biology. This method solves the functional equations of different types, and the advantage of 
this method is that it solves a problem at the direct scheme, the solution is obtained as a series sounds fast converging. We consider the operator equation $F u=g$, when $F$ is the operator represents a general nonlinear ordinary differential and $G$ is a given function. The linear part of $F$ can be decomposed into $L+R, L$ is easily invertible and $R$ is the remainder of $F$. It is therefore assumed that the nonlinear problem can be written as

$$
L z+R z+N z=g,
$$

where $N$ represents the nonlinear terms ( $N$ is a nonlinear operator). $L:$ is invertible ( $L$ is the derivative highest for what is supposed to be invertible). $R$ is a linear differential operator (of the order of less than $L$ ) and $g$ is the source term.

It can be written

$$
L u=g-R u-N u .
$$

Since $L$ is invertible we also have

$$
u=a+L^{-1} g-L^{-1}(R u)-L^{-1}(N u),
$$

where $a$ is the solution of the homogeneous equation $L u=0$, with initial conditions. The decomposition of the nonlinear term $\mathrm{Nu}$, and to do so, Adomian developed a very elegant technique as follows. We define the parameter $\lambda$ decomposition, then $N(u)$ is a function of $\lambda, u_{0}, u_{1}, \cdots$, next expansion $N(u)$ Maclurian series from $\lambda$.

We have $u$ in the form of a series,

$$
u=\sum_{n=0}^{\infty} u_{n} .
$$

We decompose the nonlinear term, $N$ as a series of special polynomials called Adomian polynomials,

$$
N u=\sum_{n=0}^{\infty} A_{n} .
$$

These polynomials are obtained by introducing a parameter $\lambda$ and writing,

$$
u(\lambda)=\sum_{k=0}^{\infty} \lambda^{k} u_{k}
$$

we deduce that

$$
A_{n}=\frac{1}{n !}\left[\frac{\mathrm{d}^{n}}{\mathrm{~d} \lambda^{n}} N u(\lambda)\right]_{\lambda=0},
$$

we have:

$$
\sum_{n=0}^{\infty} u_{n}=u_{0}-L^{-1} R \sum_{k=0}^{\infty} u_{n}-L^{-1} \sum_{n=0}^{\infty} A_{n} .
$$

To determine the $u_{n}$ we can use the following method,

$$
\left\{\begin{array}{l}
u_{0}=a+L^{-1} g \\
u_{1}=-L^{-1} R u_{0}-L^{-1} A_{0} \\
\vdots \\
u_{n+1}=-L^{-1} R u_{n}-L^{-1} A_{n}
\end{array}\right.
$$

Then

$$
u_{n}=-L^{-1} R\left(u_{n-1}\right)-L^{-1}\left(A_{n-1}\right), n \geq 1 .
$$

Finally a solution is given as

$$
\Phi_{N}(x)=\sum_{n=0}^{N-1} u_{n}(x), N \geq 1 .
$$

The exact solution is

$$
u(x)=\lim _{N \rightarrow \infty} \Phi_{N} .
$$

\subsection{The Resolution to the System with Adomian Decomposition Method}

It has a direct application of the Adomian decomposition method system. We note that the system is a more general homogeneous system of ordinary differential equations where the nonlinear term is the product of two variables. We consider the general form of a system of differential equations given as follows.

$$
X_{i}^{\prime}=\sum_{j=1}^{\infty} a_{i j} X_{i j}+\sum_{p=1}^{n} \sum_{q=1}^{n} a_{i p q} X_{p} X_{q}, i=1,2,3, \cdots, n .
$$

We can write the system of equations above as operator with $N_{i}$ is the first nonlinear term and $R_{i}$ the second term is linear, $L$ differential operator $\left(\frac{\mathrm{d}(\cdot)}{\mathrm{d} t}\right)$.

$$
L X_{i}=N_{i}+R_{i}, i=1,2,3, \cdots, n .
$$

With applying the differential operator inverse $L^{-1}$ we have

$$
X_{i}(t)=X_{i}\left(t^{*}\right)+L^{-1} N_{i}+L^{-1} R_{i}, i=1,2,3, \cdots, n .
$$

The solution $X_{i}(t)$ is given as

$$
X_{i}(t)=\sum_{m=0}^{\infty} X_{i m}(t), i=1,2,3, \cdots, n .
$$

The first nonlinear term is

$$
N_{i}=\sum_{j=1}^{n} \sum_{m=0}^{\infty} a_{i j} X_{i m} .
$$

With applying the differential operator inverse $L^{-1}$ we have

$$
L^{-1} N_{i}=\sum_{j=1}^{n} \sum_{m=0}^{\infty} a_{i j} \int_{t^{*}}^{t} X_{i m} \mathrm{~d} t, i=1,2,3, \cdots, n .
$$

The first linear term is 


$$
R_{i}=\sum_{p=1}^{n} \sum_{q=0}^{n} a_{i p q} \sum_{n=0}^{\infty} M_{i m, p, q} .
$$

With applying the differential operator inverse $L^{-1}$ about the Equation (18) on obtient:

$$
L^{-1} R_{i}=\sum_{p=1}^{n} \sum_{q=0}^{n} a_{i p q} \sum_{n=0}^{\infty} \int_{t^{*}}^{t} M_{i m, p, q} \mathrm{~d} t .
$$

For $i=1,2,3, \cdots, n$, we have

$$
\begin{aligned}
& \sum_{m=0}^{\infty} X_{i m}(t) \\
& =X_{i}\left(t^{*}\right)+\sum_{j=1}^{n} \sum_{m=1}^{\infty} a_{i j} \int_{t^{*}}^{t} X_{i m} \mathrm{~d} t+\sum_{p=1}^{n} \sum_{q=1}^{n} a_{i p q} \sum_{m=0}^{\infty} \int_{t^{*}}^{t} M_{i m, p, q} \mathrm{~d} t .
\end{aligned}
$$

So if we write the solution for each $i=1,2,3, \cdots, n$, as follows

$$
\begin{gathered}
X_{i 0}(t)=X_{i}\left(t^{*}\right) . \\
X_{i 1}(t)=\sum_{j=1}^{n} a_{i j} \int_{t^{*}}^{t} X_{i 0} \mathrm{~d} t+\sum_{p=1}^{n} \sum_{q=1}^{n} a_{i p q} \int_{t^{*}}^{t} M_{i 0, p, q} \mathrm{~d} t . \\
X_{i 2}(t)=\sum_{j=1}^{n} a_{i j} \int_{t^{*}}^{t} X_{i 1} \mathrm{~d} t+\sum_{p=1}^{n} \sum_{q=1}^{n} a_{i p q} \int_{t^{*}}^{t} M_{i 1, p, q} \mathrm{~d} t . \\
\vdots \\
X_{i,(m+1)}(t)=\sum_{j=1}^{n} a_{i j} \int_{t^{*}}^{t} X_{i, m} \mathrm{~d} t+\sum_{p=1}^{n} \sum_{q=1}^{n} a_{i p q} \int_{t^{*}}^{t} M_{i, m, p, q} \mathrm{~d} t .
\end{gathered}
$$

With applying the Adomian polynomial and then the general solution, is defined as follows $\left(t-t^{*}\right) \geq 0$,

$$
X_{i}(t)=\sum_{m=0}^{\infty} d_{i m} \frac{\left(t-t^{*}\right)^{m}}{m !}, i=1,2,3, \cdots, n .
$$

Solutions $d_{i m}$ are given as follows:

$$
d_{i 0}=X_{i}\left(t^{*}\right)
$$

$$
\begin{aligned}
d_{i m}= & \sum_{j=1}^{n} a_{i j} d_{j(m-1)} \\
& +(m-1) ! \sum_{p=1}^{n} \sum_{q=0}^{n} \sum_{k=0}^{m-1} a_{i p q} \frac{d_{q k}}{k !} \frac{d_{p(m-k-1)}}{k !(m-k-1) !}, \quad m \geq 1 .
\end{aligned}
$$

\subsection{Solve the System Using the Method (MADM)}

The solution explicite

$$
S=\sum_{m=0}^{\infty} a_{m} \frac{\left(t-t^{*}\right)^{m}}{m !}
$$

$$
\begin{gathered}
I=\sum_{m=0}^{\infty} b_{m} \frac{\left(t-t^{*}\right)^{m}}{m !} \\
Q=\sum_{m=0}^{\infty} c_{m} \frac{\left(t-t^{*}\right)^{m}}{m !}
\end{gathered}
$$

The coefficients are given with relations reccurence as follows

$$
\begin{gathered}
a_{0}=S\left(t^{*}\right), b_{0}=I\left(t^{*}\right), c_{0}=Q\left(t^{*}\right), m \geq 1 . \\
a_{m}=\mu-\mu_{1} a_{(m-1)}+\gamma b_{(m-1)} \mathrm{e}^{-\mu_{4} \tau} \\
-\alpha(m-1) ! \sum_{k=0}^{m-1} \frac{a_{k} b_{(m-k-1)}}{k !(m-k-1) !} \\
b_{m}=\alpha(m-1) ! \sum_{k=0}^{m-1} \frac{a_{k} b_{(m-k-1)}}{k !(m-k-1) !}-\left(\mu_{2}+\beta+\gamma\right) b_{(m-1)} \\
c_{m}=\beta b_{(m-1)}-\left(\rho+\mu_{3}\right) c_{(m-1)}
\end{gathered}
$$

\section{Conclusion}

This paper examined the asymptotic stability of the disease-free equilibrium and endemic equilibrium equation. If $R_{0}<1$, we proved that the disease-free equilibrium is globally asymptotically stable for any delay time, and if $R_{0}>1$, it was proved that the endemic equilibrium is zero, and disease-free equilibrium becomes unstable. We also derived two sufficient conditions for local asymptotic stability of the endemic equilibrium and sufficient condition for asymptotic stability. We resolve the system with the the adomian decompostion method.

\section{REFERENCES}

[1] R. M. Anderson, et al., "A Preliminary Study of the Transmission Dynamics of the Human Immunodeficiency Virus (HIV), the Causative Agent of AIDS," Mathematical Medicine and Biology, Vol. 3, No. 4, 1986, Pp. 229263. doi:10.1093/imammb/3.4.229

[2] Y. Asif and K. Dogan, "A Numerical Comparison for Coupled Boussines Equations by Using the ADM," Proceedings of Dynamical Systems and Applications, 5-10 July 2004, Antalya, pp. 730-736.

[3] N. T. J. Bailley, "Some Stochastic Models for Small Epidemics in Large Population,” Applied Statistics, Vol. 13, No. 1, 1964, pp. 9-19. doi:10.2307/2985218

[4] N. T. J. Bailley, "The Mathematical Theory of Infection Diseases and Its Application,” Applied Statistics, Vol. 26, No. 1, 1977, pp. 85-87. doi:10.2307/2346882

[5] M. S. Bartlett, "An Introduction to Stochastic Processes," 3rd Edition, Cambridge University Press, Cambridge, 1978.

[6] B. Batiha, M. S. M. Noorani and I. Hashim, "Numerical 
Solutions of the Nonlinear Integro-Differential Equations," International Journal of Open Problems in Computer Science, Vol. 1, No. 1, 2008, pp. 34-42.

[7] D. J. Evansa and K. R. Raslan, "The Adomian Decompositio Methode for Solving Delay Differential Equation," International Journal of Computer Mathematics, 2004, pp. 1-6.

[8] H. A. Zedan and Al-A. Eman, "Numerical Solutions for a Generalized Ito System by Using Adomian Decomposition Method," International Journal of Mathematics and Computation, Vol. 4, No. S09, 2009, pp. 9-19.

[9] D. Kaya and Inc, "On the Solution of the Nonlinear Wave Equation by the Decomposition Method," Bulletin of the Malaysian Mathematical Sciences Society, (Second Series), Vol. 22, 1999, pp. 151-155.

[10] K. R. Raslan, “The Decomposition Methode for a HirotaSatsuma Coupled KdV Equation and a Coupled MKdV Equation,” International Journal of Computer Mathematics, Vol. 81, No. 12, 2004, pp. 1497-1505. doi:10.1080/0020716042000261405

[11] S. Pamuk, "An Application for Linear and Nonlinear Heat Equations by Adomian's Decomposition Method," Applied Mathematics and Computation, Vol. 163, No. 1, 2005, pp. 89-96. doi:10.1016/j.amc.2003.10.051

[12] T. M.-D. Syed, "On Numerical Solutions of Two-Dimensional Boussinesq Equations by Using Adomian Decomposition and He's Homotopy Perturbation Method," Applications and Applied Mathematics. An International Journal, No. 1, 2010, pp. 1-11.

[13] V. Makarov and D. Dragunov, “A Numeric-Analytical
Method for Solving the Cauchy Problem for Ordinary Diferential Equations," Applied Mathematics and Computation, 2010, pp. 1-26.

[14] L. Wu, F.-D. Zong and J.-F. Zhang, “Adomian Decomposition Method for Nonlinear Differential-Difference Equation," Communications in Theoretical Physics, Vol. 48, No. 6, 2007, pp. 983-986. doi:10.1088/0253-6102/48/6/004

[15] K. Wang, W. Wang and X. Liu, "Viral Infection Model with Periodic Lytic Immune Response,” Chaos, Solitons \& Fractals, Vol. 28, No. 1, 2006, pp. 90-99. doi:10.1016/j.chaos.2005.05.003

[16] W. Wang, "Global Behavior of an SEIRS Epidemic Model with Time Delays,” Applied Mathematics Letters, Vol. 15, No. 4, 2002, pp. 423-428. doi:10.1016/S0893-9659(01)00153-7

[17] D. Greenhalgh, Q. J. A. Khanand and F. I. Lewis, "Recurrent Epidemic Cycles in an Infectious Disease Model with a Time Delay in Loss of Vaccine Immunity," Nonlinear Analysis: Theory, Methods \& Applications, Vol. 63, No. 5-7, 2005, pp. 779-788. doi:10.1016/j.na.2004.12.018

[18] G. Li and J. Zhen, "Global Stability of a SEIR Epidemic Model with Infectious Force in Latent, Infected and Immune Period," Chaos, Solitons \& Fractals, Vol. 25, No. 5, 2005, pp. 1177-1184. doi:10.1016/j.chaos.2004.11.062

[19] Y. N. Kyrychko and K. B. Nlyuss, "Global Properties of a Delayed SIR Model with Temporary Immunity and Nonlinear Incidence Rate,” Nonlinear Analysis: Real World Applications, Vol. 6, No. 3, 2005, pp. 495-507. doi:10.1016/j.nonrwa.2004.10.001 